\title{
Il mutamento delle professioni della Medicina di Laboratorio
}

\author{
The professional changing in Laboratory Medicine
}

\author{
Piero Cappelletti ${ }^{1}$
}

Ricevuto: 20 settembre 2015 / Accettato: 25 settembre 2015 / Pubblicato online: 7 ottobre 2015

(C) Springer-Verlag Italia 2015

Riassunto Lo sviluppo delle professioni in Medicina di Laboratorio è largamente determinato dall'evoluzione scientifica, disciplinare e organizzativa che il Laboratorio Clinico ha subito soprattutto negli ultimi venticinque anni. In conseguenza delle innovazioni tecnologiche, della pressione per la diminuzione dei costi, ma anche delle nuove necessità dei pazienti, il Laboratorio Clinico ha subito trasformazioni (contestuale centralizzazione e decentralizzazione) che hanno determinato la rottura spazio-temporale del rapporto medico-paziente e l'amplificazione geometrica dell'interfaccia clinica-laboratorio con le conseguenze della moltiplicazione e complessità dei temi della sicurezza/qualità delle analisi, dell'interpretazione clinicamente adeguata e fondata e delle abilità comunicative in favore di soggetti diversi dal tradizionale medico "curante". Lo scenario attuale è dominato dallo sviluppo dell' era elettronica, avvenuto nel decennio scorso con la comparsa del "paziente digitale", della Hyperlinked Society e di ciò che è definito Health 2.0 e Medicine 2.0 (doctor Google, doctor Smartphone e Wearable Health Technology).

Dettato dai cambiamenti intervenuti, il mutamento professionale è stato imponente: crescita professionale e legislativa dei tecnici di laboratorio, prevalente lavoro all'interfaccia dei dirigenti con maggiori accenti per la specializzazione tecnica o il rapporto clinico, necessità di aumentare le competenze in particolare comunicative. In questo quadro la Società Scientifica ha prodotto un'elaborazione concettuale che prevede: (1) la necessità di una nuova alleanza tra le professioni per la creazione di un'equipe multi-disciplinare e multi-professionale articolata nelle sue autonomie e responsabilità con attività collaborativa e coordinata tenuta insie-

$凶$ P. Cappelletti pie.cappelletti@gmail.com

1 SIPMeL, Castelfranco Veneto, TV, Italia me dalla mission del laboratorio e dalla leadership clinicoorganizzativa; (2) la necessaria mobilità dei "ruoli" con il superamento dei confini rigidamente stabiliti per una migliore collaborazione interna e un migliore servizio al cittadino; (3) la necessaria modifica delle competenze, il loro proliferare e la loro specializzazione, insieme con la padronanza di alcune abilità comuni. Oggi, nell'era elettronica e della complessità, di fronte all'espansione dell'interfaccia clinica-laboratorio, alla moltiplicazione degli utenti (medici di medicina generale, medici specialisti, professionisti sanitari, altri professionisti, stakeholders, pazienti, cittadini) e alle problematiche del paziente digitale e del paziente complesso, i compiti dei professionisti della Medicina di Laboratorio sono moltiplicati e complicati: accanto a quelli tradizionali analitici e interpretativi, tipici del "laboratorio centrale", che vanno ulteriormente specializzandosi, vi sono quelli destinati a garantire qualità, sicurezza, interpretazione e appropriatezza in tutti i punti della rete extra-ospedaliera di attività, compresi quelli “digitali”, quelli destinati a garantire innovazione ed "evidenze", quelli destinati a realizzare le "trasformazioni" organizzative e quelli necessari al ruolo informativo/formativo della Medicina di Laboratorio. Solo un' equipe coordinata e collaborativa ricca delle sue specificazioni ma tenuta insieme dalla mission e dalla leadership può garantire un'adeguata risposta alla sfida.

Parole chiave Medicina di Laboratorio - Professioni . Complessità · Medicina 2.0

Summary The professional changing in the Laboratory Medicine is determined by the scientific, disciplinary, and organizational evolution of the Clinical Laboratory in the recent years. Technological innovations, costs pressure, and new patients' needs and requests determined a big transformation (simultaneous centralization and decentralization) 
that produced a geometrical amplification of the cliniclaboratory interface. Today, in the world of complexity and of hyperlinked society, the clinic-laboratory interface is challenged by Health and Medicine 2.0, i.e. Doctor Google, Doctor Smartphone, and the Wearable Health Technology.

The professional changing, in the recent past, was characterized by qualitative/quantitative transformation of contents and performances of technologists profession, by increased activity at clinic-laboratory interface with more technic and/or clinical competences for consultants, and by new competences including communicative ones for all. The Society elaborated some main concepts about transformation of professions: the role of multidisciplinary and multiprofessional team; the moving boundaries of "roles" and "professions"; the development of new, specific or shared, competences to answer to digital patient and complex patient. Today, in a new way, the Laboratory Medicine Team continue to warrant quality and safety, appropriateness and interpretation of laboratory medicine in all points of the expanded, complex, and digital clinic-laboratory interface; to warrant implementation of innovations for an up-to-date and effective laboratory medicine and the production of evidences for a evidence-based and choosing wisely laboratory medicine; to warrant its role for information and education of multiplied users (physicians, patients, stakeholders, citizens and politics) about the essentials and outcomes of our discipline and our profession.

Keywords Laboratory Medicine - Professional workforce . Complexity $\cdot$ Health and Medicine 2.0

Lo sviluppo delle professioni in Medicina di Laboratorio è largamente determinato dall'evoluzione scientifica, disciplinare e organizzativa che il Laboratorio Clinico ha subito soprattutto negli ultimi venticinque anni [1].

\section{L'evoluzione del Laboratorio Clinico e il dibattito italiano}

Alla nascita della moderna Medicina di Laboratorio, dopo la metà del secolo scorso, l'attività del Laboratorio è pensata come un percorso produttivo lineare tra l'input della richiesta e dei campioni biologici e l'output della risposta, con al centro l'attività strettamente analitica, poco visibile (black box) e compresa dall'utilizzatore clinico e la sua suddivisione in "settori" è dettata talora da motivazioni disciplinari (ematologia, microbiologia), ma spesso da motivazioni puramente tecniche (immunometria, RIA).

Negli anni Novanta il panorama organizzativo cambia drasticamente [2], in conseguenza delle innovazioni tecnologiche, in particolare le innovazioni radicali, quali i feno- meni della miniaturizzazione e dello sviluppo della information and communication technology (ICT) [3], della pressione per la diminuzione dei costi [3], ma anche delle nuove necessità dei pazienti [4].

Da un lato, l'organizzazione dei Laboratori di diagnostica urgente conosce una curva evolutiva, dalla dispersione all'interno del Laboratorio all'autonomia di un settore e quindi di nuovo all'integrazione e, contemporaneamente, la pressione per la diminuzione del Turn-around Time (TAT) e la disponibilità di adeguati supporti tecnologici spingono la diffusione di strumenti dei Point of Care Testing (POCT) [1]. D'altra parte, anche nei Laboratori "centrali" la richiesta di diagnostica in tempo reale con migliori TAT e con adeguata attenzione alla fase pre-analitica e post-analitica mostra i limiti del Laboratorio "compartimentato" e "a silo" e la necessità di un'organizzazione focalizzata al TAT con laboratori core fortemente automatizzati, Laboratori a risposta rapida e Laboratori satellite specialistici [4]. Nascono così il concetto del ri-arrangiamento dell' organizzazione lineare del lavoro nel Laboratorio per fasi (pre-analitica, analitica e postanalitica) [2], l'opzione del ri-assemblamento delle dotazioni tecniche, separando talora le convenienze analitiche dalle competenze disciplinari, nella logica pianificatoria e gestionale di organizzazioni innovative secondo la metafora dell'organizzazione come cultura [5] e il diffondersi di una configurazione generale dei Laboratori caratterizzata dalla contemporanea centralizzazione (consolidamenti strumentali e strutturali; merging e reti geografiche) e decentralizzazione (analisi decentrate come POCT, NPT e self-testing) delle attività [6]. Queste innovazioni hanno determinato, in generale, la rottura spazio-temporale del rapporto medico-paziente e, per la Medicina di Laboratorio, l'amplificazione geometrica dell'interfaccia clinica-laboratorio con le conseguenze della moltiplicazione e complessità dei temi della sicurezza/qualità delle analisi, dell'interpretazione clinicamente adeguata e fondata e delle abilità comunicative in favore di soggetti diversi dal tradizionale medico "curante". La rottura dei rapporti e delle competenze tradizionali consente il superamento di organizzazioni centrate sulla tecnologia e sulle abitudini della workforce per una vera organizzazione sanitaria disegnata sulle necessità del paziente [6].

Questi temi sono all'attenzione della nostra Società scientifica da più di dieci anni e sono stati affrontati dapprima per comprendere le caratteristiche del mutamento e ribadire i fondamenti della disciplina (Congresso di Modena 2004 sulle trasformazioni organizzative e professionali, Congresso di Trieste 2005 sull'essenza della disciplina, Congresso di Palermo 2006 sulla sicurezza altra faccia della qualità, Congresso di Rimini 2008 sul cambiamento, Congresso di Riva 2007 sulla Medicina di Laboratorio come servizio essenziale, prospettiva interdisciplinare e visione olistica), poi per caratterizzare la Società e la disciplina rispetto alle innovazioni diagnostiche legate ai nuovi biomarcatori e alla Medicina Traslazionale, alle omiche e alla 
Medicina Personalizzata (Congresso di Napoli 2009 sulla Medicina Personalizzata, Congresso di Verona 2010 sulla 4Ps Medicine, Congresso di Torino 2013 su Innovazioni ed Evidenze, Congresso di Rimini 2014 sulla Complessità). Il quadro più recente è stato descritto da alcuni editoriali e articoli de "La Rivista Italiana della Medicina di Laboratorio": nell' ordine concettuale, "Medicina di Laboratorio: un futuro possibile" [6] che ripercorre l'evoluzione e offre prospettive anche visionarie [7], "Scenari e ruoli al tempo della crisi" (tratti dalle relazioni di apertura del Congresso di Salerno) [5, 6, 8-11] sulle origini del cambiamento e sull'evoluzione dei ruoli e "Innovazione organizzativa" [4] sull'impatto della tecnologia sull'organizzazione e sulle prospettive del servizio della Medicina di Laboratorio.

I punti focali di questa elaborazione concettuale, per il tema qui affrontato del mutamento delle professioni, sono in estrema sintesi i seguenti. Per rispondere adeguatamente alla sua natura ("fornire informazioni cliniche"), la Medicina di Laboratorio deve organizzarsi in una "rete" ("micro" nei rapporti interprofessionali e interdisciplinari dell' equipe e "macro" nei rapporti tra organizzazioni che servono la popolazione "naturale") gestita (gerarchica e differenziata: non si tratta di una rete da pescatore ma di una tela di ragno) di servizi clinici (non semplicemente prestazioni o esami), per governare l'intero processo che la origina, attiva e utilizza [2]. Il punto chiave diventa l'interfaccia clinicalaboratorio, amplificata dalla struttura a rete, rivolta al clinico e al paziente, coinvolgente tutta l'equipe, caratterizzata dalla qualità della comunicazione e dalla sua coerenza con l'obiettivo clinico. I due aspetti, organizzativo e concettuale, vanno fusi in un approccio globale, dove il servizio della Medicina di Laboratorio e il suo impatto sulla cura dei pazienti vengono declinati nella rete dei rapporti che ne costituiscono la popolazione "naturale", secondo modalità congrue al concetto di centralità del paziente e di appropriatezza dell'intervento [2].

Rispetto al dibattito aperto da SIMeL sui temi organizzativi (Congresso di Modena 2004; Congresso di Salerno 2012) [8], la discussione italiana sulla centralizzazione/decentralizzazione delle attività di Laboratorio si è incentrata prevalentemente sulle concentrazioni e principalmente sulle caratteristiche dei merging e dei network e sulle problematicità relative, spesso con accenti parziali e riduttivi come la querelle sugli "esamifici" o la discussione sulla collocazione ospedaliera/extra-ospedaliera dei Laboratori territoriali [12], raramente con aperture al futuro quali la ristrutturazione dei flussi operativi [13], la rivoluzione informatica [14], la riconfigurazione delle discipline [5], il riallineamento delle specialità diagnostiche [15] (Laboratorio lato sensu e diagnostica per immagini) connesso al timeline trending. Anche il recente fenomeno Theranos [16]-35 analiti in una goccia di sangue a prezzi imbattibili-s'inserisce in realtà nella scia delle concentrazioni e dell'outsourcing e come tale va affrontato. D'altra parte, gli allarmi per il taglio degli addetti, conseguente alle concentrazioni, va colto con cautela: è ben vero che nel decennio 2000-2010 si può stimare che sia stato perso circa il $30 \%$ di dirigenti di Laboratorio in Italia, ma il numero di dirigenti per numero di abitanti è ancora di 103/milione contro una media europea di 77,5/milione (più di noi solo Grecia, Cipro, Francia, Portogallo, Estonia e Romania), secondo il questionario europeo [17] pubblicato nel 2012.

La discussione sulla decentralizzazione è stata ancora più provinciale. Mentre molti hanno enfatizzato esclusivamente i limiti strumentali dei POCT, che certamente ci sono, SIMeL ha, fin dal Convegno di Vicenza del 2001 [18], esaminato vantaggi e svantaggi, opportunità e rischi della decentralizzazione con animo laico e avendo ben presente che si tratta comunque di una "diversa modalità di declinazione della Medicina di Laboratorio" che deve essere presidiata [19]. Ciò che sfugge ai più è che la previsione di Robert Felder et al [20] sul fatto che nel 2020 1'85\% dei test sarà effettuato fuori dal Laboratorio appare azzardata se si considera solo il POCT ospedaliero o para-ospedaliera (ancora oggi il 55\% dei test è effettuato nel Laboratorio Centrale) [6], ma è invece azzeccata se teniamo conto dei test effettuati dai medici extra-ospedalieri (in particolare nei Paesi in via di sviluppo) e soprattutto del self-testing in espansione dilagante nei Paesi sviluppati, a cominciare dal più antico test di gravidanza, passando per i monitoraggi dei pazienti cronici e la diagnostica rapida delle malattie infettive, per finire ai test genetici. Ovviamente ciò pone gravi problemi di qualità/sicurezza dei test e d'interpretazione degli stessi e più in generale di informazione/formazione della popolazione. Sotto questo profilo, il "compito sociale" della Medicina di Laboratorio è stato descritto da SIMeL ancora nel "Patto per la Modernizzazione ed Umanizzazione della Medicina di Laboratorio" [21].

\section{L'era elettronica}

Lo scenario attuale è dominato dallo sviluppo dell'era elettronica, avvenuto nel decennio scorso con l'esplosione del "paziente digitale" e la nascita di ciò che è definito Health 2.0 e Medicine 2.0 nella Hyperlinked Society [22]. Le definizioni non sono nette; tuttavia Van De Belt et al [23] sostengono che questi termini "2.0" differiscono dal vecchio concetto di $e$-Health nella loro enfasi sul social networking tra pazienti e pazienti (blog, social network) e pazientifornitori clinici. Medicine 2.0 starebbe a significare l'uso del 2.0 da parte dei professionisti della Medicina (utilizzo clinico), mentre Health 2.0 dovrebbe, viceversa, fare riferimento alle applicazioni per i pazienti, non esclusivamente cliniche ma in generale di self-tracking. 
In principio fu "Doctor Google" (il paziente consulta Internet per diagnosi, prognosi, terapia e altre notizie su sintomi e segni auto-riconosciuti o su timori generici) e già alla fine degli anni Novanta se ne indicavano opportunità e rischi in relazione alla salute individuale e al rapporto medicopaziente [24]. Secondo Health Online 2013 del PEW Research Center [25] il 35\% degli americani usa normalmente Google a scopi medici, un terzo di questi non chiede conferma dei risultati al suo medico (dieci anni prima era il $16 \%$, cioè la metà), dei due terzi che ne parlano con il proprio medico il $41 \%$ ha conferma delle ipotesi diagnosticoterapeutiche trovate, ma il $18 \%$ ha una diagnosi/terapia diversa. Si crea, inoltre, una nuova forma patologica la "cyberchondria", possibile campo di azione di servizi di rete profilati ad hoc.

Quindi, alla metà del decennio scorso esplode l'uso dei social media a fini sanitari [26]: Health 2.0 is a more patientcentred participatory model of healthcare, emphasizing online (public or private) interactivity between consumers, health providers and librarians using social networks ... and an ability to share medical evidence, patient data and anecdote to improve health outcomes on a global scale [27]. I siti più popolari di web 2.0 sono Facebook, Flickr, Twitter, Wikipedia e YouTube. Secondo molti autori Health 2.0 comporta partecipazione ed equità ed è un mezzo potente di patient-empowerment. Hughes et al [27] indicano, però, 4 criticità nella health/medicine 2.0: carenza di chiare definizioni; perdita del controllo medico sulle informazioni; pericolo di conseguenze dannose sulla salute; problemi di proprietà e di privacy. Ma il cambiamento non si arresta.

Il passo seguente è la comparsa e l'affermarsi di "Doctor Smartphone" [28]. L'85\% degli americani ha uno smartpho$n e$; per il $54 \%$ di loro la prima ragione per l'uso di app è il controllo di salute (health monitoring); il $76 \%$ è convinto che l'uso delle app migliorerà la sua salute; l' $82 \%$ preferirebbe un consulto con il proprio medico via smartphone (52\% con video) e controlla la prescrizione sullo smartpho$n e$ per decidere se seguirla o meno [25, 29, 30]. Solo nel secondo semestre del 2014 l'aumento nell'uso di app sanitarie (fitness e salute) è stato del 62\% [29]. Oggi lo smartphone rappresenta, oltre all'uso di app per self-tracking, un mezzo di controllo per i pazienti (parametri vitali con iOS8, conduttanza cutanea con iWatch, ECG con iPhone, Power sleep, Ovia pregnancy, Breast cancer diagnosis grade, uCheck Biosite e Sensori per glucosio, Qloud lab per coagulazione, Alb urinaria, Anemia Check ecc.) [29-31], ma anche uno strumento per i medici (app con riprodotti testi, app per diagnosi come Isabel e terapia come Epocrates, Ascd Risk estimator, Sm@rtADI per assistenza domiciliare, Filmic Pro per fundus oculi, Opteractive per esame vista, Fotografie di ferite e di preparati microscopici, Microfotografie ecc.) [29-31].

Associata all'uso di Internet e Smartphone si apre la nuova frontiera del "Wearable" [31, 32]: cardiofrequenzimetri, gilet per misura pressione arteriosa, micro-pacemaker senza fili, siringa defibrillante per aritmie, fascia Interaxon MUSE per onde cerebrali, Lenti smart che misurano la pressione intraoculare per glaucoma, Lenti bioniche per realtà aumentata, Google glass per fotografare lesioni, Spirale sensore per glucosio su palpebre, Aquaris apparecchio acustico impermeabile, Sciarpa per ipovedenti, fascia Bodymetric Core 2 per parametri biometrici, cintura Lumaback per postura, Intimo intelligente che misura composizione sudore, Bilancia per peso, massa e distribuzione, MINO body per neonato misura parametri locali ecc. Le nuove applicazioni sono fertile terreno per start-up e sono descritte sulla stampa finanziaria e su quella popolare ancora prima che su quella scientifica. Sul "Corriere Economia" del 14 settembre un articolo [33], intitolato "Internet delle cose. Così i piccoli possono diventare grandi", ha come sottotitolo "I big dell'hi-tech a caccia di start-up e PMI innovative" e come occhiello "Già otto milioni gli oggetti connessi in Italia per un giro d'affari di 1,5 miliardi. Destinati a crescere velocemente...". I casi italiani illustrati riguardano la medicina connessa: EKO CORE stetoscopio digitale; AMYKO braccialetto smart controlla e memorizza dati vitali come pressione arteriosa; DERMOPACHT cerotto elettronico per diffusione medicinali sotocute; BLOOD GLUCOSE MONITOR connesso tramite smartphone a un portale medico (quest'ultima applicazione già approvata FDA). La FDA (Food and Drug Administration) [34], infatti, è già intervenuta per dettare regole di approvazione e comportamentali della Medicine 2.0.

L'autocontrollo di molti parametri biologici attraverso dispositivi elettronici portatili (unplugged medicine) è destinato a segnare una discontinuità epocale nella gestione della salute [35].

\section{La trasformazione delle professioni}

Dettato dai cambiamenti intervenuti, il mutamento professionale è stato imponente. All'inizio del cambiamento (le concentrazioni) le possibilità evolutive delle professioni sono state ben illustrate da Wood [36] sull'evoluzione di responsabilità organizzative e di qualità/sicurezza dei TSLB (Tecnici sanitari di Laboratorio Biomedico), da Diamandis [37] sul valore specialistico ( $M r / M s$ Something) e di ricerca dei DSLB (Dirigenti Specialisti di Laboratorio Biomedico) e dal Royal College of Pathologists [38] sull'impostazione clinica e di lavoro all'interfaccia dei medici patologi.

I tecnici sanitari di laboratorio biomedico hanno attraversato un passaggio impegnativo ma esaltante, costituito dalle novità formative (laurea) e professionali (riconoscimento giuridico e ordine) che aprono nuovi scenari. Molti devono, però, mutare e rinnovare le proprie abilità in rapporto alle 
ristrutturazioni organizzative, ma per tutti si apre il compito di garanti della fase analitica, del suo controllo e dei suoi risultati e per quelli incaricati di coordinamento (di "isola", di funzione, dei POCT, del LIS, del controllo di gestione, di unità operativa, di dipartimento) il compito di controllori organizzativi e gestionali dei processi. Per tutti si apre la necessità dell'aumento delle competenze comunicative e di relazione con gli altri attori professionali del mutamento della sanità [2].

Per i dirigenti, nel rispetto delle specificità professionali, il focus si è progressivamente spostato dalla fase analitica della grande routine all'interfaccia clinica-laboratorio e alla partecipazione diretta all'attività epidemiologica e clinica. Non si tratta certo di dimenticare gli aspetti analitici e il loro ruolo di fondamenta sulle quali si costruisce la piramide del sapere e dell'agire della Medicina di Laboratorio, ma di accettare l'espansione di ruolo che il nuovo comporta. In area anglosassone le caratteristiche degli "scientist" sono state delineate da Diamandis [37], seppure nella pluralità dell'accezione per laurea, obiettivi e tipo di lavoro, in riferimento ai compiti di servizio diagnostico, educazione professionale e ricerca. Particolarmente interessante, nello scritto di Diamandis, sono le "regole del successo" che egli consiglia ai giovani biochimici clinici e che sono articolate nelle "Nobody rules", cioè ciò che ciascun professionista deve conquistarsi perché nessuno gli concederà spontaneamente (spazio e tempo per la ricerca, ruolo nell'educazione e la scrittura, ambizione a "distinguersi"), e nelle "Success rules" definite dalle attitudini, dall' apprendimento continuo, dall'esperienza e in particolare dall'ambizione di diventare "Mister or Madam Something" cioè della specializzazione anche di nicchia riconosciuta dai clienti clinici.

Nel 2004, il Royal College of Pathologists [38] ha prodotto un documento, la cui revisione è attesa per il novembre di quest'anno, in cui ha provato a immaginare i futuri ruoli dei medici di laboratorio e nel 2006 l'Academy of Clinical Laboratory Physicians and Scientists [39] ha proposto un curriculum che definisce gli obiettivi, le competenze e il modo di valutarle del patologo. Il medico di laboratorio e il patologo devono essere in grado di comunicare come consulenti medici verso clinici e pazienti, così come di gestire al meglio l'impresa del laboratorio, conoscendo scienza e tecnologia e assicurando la qualità, l'appropriatezza e l'utilità dei dati. Il patologo è innanzitutto un clinico, ma deve conoscere metodi e utilizzo dei dati analitici e la loro gestione informatica, nonché le implicazioni terapeutiche per le quali esercita il ruolo di consulente. Deve comprendere il ruolo della ricerca nella sua più ampia definizione di "decision-making", sviluppo dei test, generazione di conoscenza e di educazione continua. I consultant medici lavorano e sono spesso leader di team multiprofessionali in partnership con personale non medico che ha delegate specifiche responsabilità. Le competenze comuni sono legate alla cura dei pazienti, alla conoscenza medica, all'apprendimento basato sulla pratica, alle abilità interpersonali e di comunicazione, alla professionalità (attitudine al lavoro, interazione personale, responsabilità, regole ed eccellenza) e alla pratica dei sistemi organizzativi e regolatori. Le competenze specifiche, articolate per livelli, riguardano la chimica, il POCT, l'ematologia, la microbiologia, la medicina trasfusionale, l'immunologia e l'immunogenetica, la patologia molecolare (compresa la citogenetica), la gestione di laboratorio, l'informatica. Per la valutazione delle competenze sono proposti diversi metodi dai questionari all'esame scritto, dalle simulazioni al caso clinico, dalle "checklist" al " $360^{\circ}$ global rating". Le singole carriere sono certamente diversificate e non tutti i dirigenti posseggono tutte le competenze, ma il curriculum è stato proposto per cogliere le conoscenze ed abilità core della disciplina.

Nel 2005 un gruppo di lavoro della European Cотmunities Confederation of Clinical Chemistry and Laboratory Medicine (EC4) [40] ha pubblicato una guida per definire le competenze richieste a un "consultant" in chimica clinica e Medicina di Laboratorio che identifica un modello di attività specifiche comprendente la pratica clinica (segnalazioni e interpretazioni), la qualità delle prestazioni, la responsabilità professionale, la formazione e la ricerca, la gestione. L'elenco delle ottantasei competenze richieste, espresse come semplici generici standard di abilità, si articola in sei grandi aree: clinica, scientifica, tecnica, comunicativa, gestionale e di leadership, professionale (autonomia e responsabilità). L'aspetto più innovativo è rappresentato dalle "competenze necessarie per comunicare efficacemente all'interno della disciplina, con gli utenti e con la più ampia comunità clinica, scientifica e politica", comprendenti: le capacità di usare la "information technology" e i moderni metodi di comunicazione e di capire le richieste di back-up, sicurezza e regolazione per l'uso legale ed etico dell'informazione, la comprensione dei benefici dell'information technology avanzata come mezzo di migliore comunicazione utilizzando codici unificati e reti elettroniche, la capacità di guidare un gruppo di discussione e moderare un incontro di comitato efficacemente, di presentare risultati in modo scritto e orale tramite relazioni, lavori scientifici, poster, seminari, tutoraggi e letture, di addestrare colleghi in aree definite, di comunicare efficacemente con i colleghi all'interno della disciplina e con gli utenti del servizio, di comunicare i risultati e le necessità della disciplina ai dirigenti aziendali e alla più vasta comunità clinica, la consapevolezza delle opportunità e limiti della promozione della disciplina nelle più ampie arene pubbliche e politiche, la capacità di rappresentare la disciplina negli incontri multidisciplinari e operativi per la pianificazione strategica, l'esperienza di audit clinici esterni come mezzo per migliorare sia la qualità del servizio che l'appropriatezza dei test, la consapevolezza dell'importanza del lavoro di squadra 
nello sviluppo della disciplina e della professione, la partecipazione attiva negli organismi professionali importanti e negli incontri multidisciplinari scientifici e clinici.

Se le colonne portanti del tempio della qualità in Medicina di Laboratorio sono il fondamento etico, la metodologia scientifica, la razionalità gestionale e la valorizzazione delle risorse umane, il basamento su cui tutte poggiano è la comunicazione.

Questi concetti furono ripresi, alla luce della situazione italiana, in "Preparando Modena: cos'è e dove va la Medicina di Laboratorio" [2], "La risposta della Medicina di Laboratorio al quesito clinico" [41] e "Come cambia la Medicina di Laboratorio" [42], con tre importanti riflessioni originali:

(1) la necessità di una nuova alleanza tra le professioni per la creazione di un'equipe multi-disciplinare e multiprofessionale articolata nelle sue autonomie e responsabilità con attività collaborativa e coordinata tenuta insieme dalla mission del Laboratorio e dalla leadership clinico-organizzativa [1] (questa intuizione-che distingue SIMeL-SIPMeL da altre Società e che distingue la Società scientifica da altre organizzazioni sindacali o professionali-maturerà lungo il decennio per approdare alla consacrazione statutaria nel 2014);

(2) la necessità di mobilità dei "ruoli" delle diverse professioni di laboratorio con il superamento dei confini rigidamente stabiliti per una migliore collaborazione interna e un migliore servizio al cittadino [4];

(3) la necessaria modifica delle competenze, il proliferare e la loro specializzazione, insieme con la padronanza di alcune abilità comuni tra le quali primeggia, nuova, quella comunicativa $[4,41]$.

In questo difficile momento di cambiamento, le competenze delle professioni della Medicina di Laboratorio si ridefiniscono in una visione comune e prospettica, definita dagli obiettivi dell' organizzazione e trainata dalle indicazioni della leadership [4]. Emerge il concetto di "ruolo" nell'accezione di un'istituzionalizzazione provvisoria e contingente delle soluzioni che gli attori trovano al problema della cooperazione e di spazio d'azione della persona nell'insieme di relazioni che costituiscono l'organizzazione. Ciò pone il tema dei confini e dei contenuti dei ruoli: nell'organizzazione innovativa i confini sono mobili e da ridefinire e i contenuti sono uno spazio discrezionale che comporta rischi, incertezza, comunicazione, negoziazione. È necessario agire sulle competenze per stabilizzare gli aspetti oggettivi del ruolo: capire e chiarire il mandato, definire i legami organizzativi, stabilizzare lo status; valutare gli aspetti soggettivi: obiettivi, visione personale, motivazioni, competenze e sviluppo; gestire i rapporti con gli altri ruoli: operare su interessi e problemi, comunicare la vision, orientarsi al futuro, perseguire successi, costruire coalizioni.

Diviene, quindi, centrale il tema delle competenze: la competenza è una caratteristica delle persone che consente performance eccellenti (risultati misurabili) e che dipende da abilità, conoscenze, immagine di sé, attitudini e motivazioni progressivamente più profonde (modello iceberg). Secondo il modello di Weggeman rivisto da Goldschmidt [43], la competenza è il prodotto dell'informazione per esperienza, abilità, attitudini e in Medicina di Laboratorio si appalesa nei crinali tra risultati e informazione e tra informazione e decisione. La complessità delle definizioni rivela la complessità del tema: competencies (USA) alla ricerca dell'eccellenza (best performers), via metodo induttivo sperimentale; competences (UK) per migliorare gli standard minimi delle performance; competence $(\mathrm{F})$ abilità riconosciuta, in atto, in situazione, finalizzata per la modifica dell'organizzazione; competenza come sense making meta-situazionale. Un problema complesso, infine, è la valutazione delle competenze, che il gruppo di lavoro EC4 affida alla preparazione professionale accademica, al riconoscimento di corpi professionali indipendenti (registration), allo sviluppo professionale continuo e alla revisione delle prestazioni (performance review) come parte dello sviluppo professionale continuo o come valutazione formale delle competenze individuali con prove di abilità (proficiency standard). Lo sviluppo delle competenze, d'altra parte, non si ottiene solo con la formazione, ma con la personalizzazione e responsabilizzazione (studio del gap e taylorizzazione della formazione). Il leader ha un ruolo centrale nello sviluppo delle competenze (ruolo trasformazionale) necessarie alla trasformazione del Laboratorio.

Un tema ancora irrisolto è l'armonizzazione delle conoscenze e delle competenze degli specialisti in Medicina di Laboratorio, almeno all'interno dell'Unione Europea, anche alla luce delle differenze nel nome, background culturale, organizzazione delle sub-specialità, lunghezza e contenuto della formazione, responsabilità professionali, requisiti per il riconoscimento professionale e conferma dello stesso in ambito UE [17]. In un recente editoriale [44] Graham Beastall riconosce che la strada dell'armonizzazione è piuttosto lunga e che le prospettive future credibili sono relative non tanto a un'irrealistica unificazione dei contenuti e delle pratiche della specialità e delle professioni mediche e non, ma alla messa a punto di standard e formazioni che costituiscono un quadro di insieme per ridurre la variabilità degli approcci delle diverse sub-specialità e dei diversi contesti socio-professionali. Altri punti da sviluppare in un'ottica di armonizzazione sono il ruolo della leadership delle equipe di laboratorio che deve essere orientata alla clinica e le caratteristiche del continuing professional development (CPD) tale da consentire non solo l'aggiornamento delle conoscenze, ma un'armonizzazione nello sviluppo delle competenze. Come si vede, da altra angolazione culturale si giunge nei pressi delle elaborazioni delle nostra Società.

All'inizio del secondo decennio di questo secolo, il mutamento dei ruoli e delle competenze indicate come necessari, 
"ai tempi della crisi", sono ancora largamente pensati come ancorati agli effetti della concentrazione [45]. Rispetto alla ricordata ricerca bocconiana del 2012 [45], il quadro dei nuovi ruoli è stato nel Congresso di Salerno precisato in modo più ricco e complesso [6]: il ruolo di sacerdote della tecnologia comprende innovazione tecnologica oltre che selezione di tecnologie e partner, controllo di qualità, valutazione delle forme di gestione; il ruolo organizzativo-gestionale consiste nella pianificazione strategica, organizzazione e gestione del laboratorio e dei suoi addetti, con particolare attenzione ai processi, al prodotto e al loro controllo; il ruolo di consulente clinico si esplica come consulenza nella richiesta e nell'interpretazione dei casi singoli, ma anche di gruppi interdisciplinari, in particolare a fini di audit clinico; il ruolo del governo clinico si esplica nella definizione collaborativa di "evidenze", linee guida, EBLM (evidence-based laboratory medicine) e PDTA, oltre che nella formazione di specialisti e MMG.

Nel frattempo i compiti generali della Medicina di Laboratorio sono stati illustrati in "La risposta della Medicina di Laboratorio al quesito clinico" [41], riprendendo il Patto del 2004. In particolare per i rapporti con i cittadini si diceva: "Il compito dell'equipe di laboratorio verso i cittadini, dunque, è quello di fornire adeguate informazioni alla popolazione sull'uso e le modalità di accesso alla diagnostica, di formare attraverso interventi generali e specifici l'opinione pubblica rispetto al significato interpretativo e non assoluto dei risultati degli esami di laboratorio, di indirizzare correttamente a un appropriato iter terapeutico chi si rivolga direttamente al Laboratorio. Questo compito sociale enfatizza la nuova frontiera del Laboratorio, fuori dalle mura, ed è una risposta, anche se parziale, al problema così sentito della scarsa visibilità". Le trasformazioni successive (il paziente digitale) richiedono ancor più questa predisposizione.

\section{Prospettive professionali nell'era elettronica}

Oggi, nell'era elettronica, di fronte all'espansione dell'interfaccia clinica del Laboratorio, alla moltiplicazione degli utenti (MMG, medici specialisti, professionisti sanitari, altri professionisti, stakeholders, pazienti, cittadini) e alle problematiche del paziente digitale, i compiti dei professionisti della Medicina di Laboratorio sono moltiplicati e complicati.

In questo contesto, infatti, nascono nuovi ruoli e altri devono essere "inventati": clinical science manager; quality manager, risk manager, education \& training officer and practice tutor, LIS manager, POCT manager; advanced practitioner scientist, clinical diagnostic scientist, skills mix manager, audit manager, ICT manager ecc. [6].

Alcune figure professionali vanno scomparendo in conseguenza delle dinamiche del mercato del lavoro, pur essendo ancora necessarie: penso ai Dirigenti Chimici e alle opportunità offerte dalle tecnologie per diagnostiche innovative come il Maldi TOF/TOF MS per la proteomica.

Infine, altre nuove figure professionali (innanzitutto informatici, bio-informatici e statistici) sono attese nell'integrazione delle equipe, necessarie per un ruolo traslazionale ed efficacemente comunicativo della Medicina di Laboratorio (translational scientist, comunication scientist).

Diviene ancora più centrale il ruolo dell'equipe, concetto che peraltro enfatizza il ruolo del leader trasformativo ( $\mathrm{cul}$ tural transformer), perché solo con un'attività coordinata $\mathrm{e}$ condivisa che valorizzi le abilità professionali specifiche ma che le riconduca a unità nel segno della mission del Laboratorio sarà possibile mantenere, nel mondo della complessità [46], i compiti tradizionali della Medicina di Laboratorio: fornire informazioni e consulenza per la salute dei pazienti/cittadini tratte da campioni biologici umani e grazie all'uso di una rete complessa di metodi e strumenti di elevata tecnologia. La diffusione extra-ospedaliera degli esami e la loro miniaturizzazione è a carico principalmente di un aumento globale dei test effettuati [6]: nei Laboratori centrali si continueranno comunque a eseguire test complessi, specialistici e tecnologicamente avanzati (diagnostica molecolare, spettrometria di massa, tecnologie producenti big data) e batterie di test correlati e sincronici per produrre diagnosi e indicazioni di terapie correlate, da un lato, e a validare nuovi metodi e biomarcatori producendo e validandone le "evidenze" [47], dall'altro.

Solo un intelligente lavoro di equipe potrà, oltre a ciò, garantire affidabilità e qualità/sicurezza dei risultati (innovazione, specializzazione, controllo) in ogni sito di produzione (lab centrale, POCT e laboratori satelliti, NPT, self testing); innovare "traslando" correttamente nuovi metodi e marcatori dalla ricerca alla cura comprendendone e, per quanto possibile, guidandone le potenzialità "trasformative" dell'organizzazione patient-centered e delle nuove necessità pratiche e di contenuto del "paziente digitale" o ancora "semidigitale" da un lato e del "paziente complesso" dall'altro [46]; a operare all'interfaccia clinica-laboratorio a fronte della sua geometrica amplificazione, producendo evidenze e declinandole (linee guida e raccomandazioni; indicazioni choosing wisely) e verificandone l'applicazione e l'appropriatezza (audit) da un lato e ampliando il proprio compito sociale informativo/formativo con l'utilizzo dei mezzi tipici del paziente digitale dall'altro.

In questa prospettiva Brain Smith [48], a fronte dei grandi cambiamenti dell'era elettronica-individuati nell'IT, nei POCT, nelle tecnologie "big data" e negli EMR (Electronic Medical Record)—sostiene che la tripla attività di laboratorio (servizio, formazione, ricerca) è ancora possibile, ma non alla vecchia maniera, bensì guardando ai vantaggi dei big data e dell'era elettronica, alla necessità di collaborazioni anche in remoto, non rifuggendo dalle responsabilità amministrative e re-immaginando i ruoli degli attori del 
team, sempre che si sia sufficientemente aperti al futuro e contemporaneamente preparati in qualche sub-specialità.

Le implicazioni a lungo termine saranno principalmente la necessità di sviluppare: la comprensione di dati globali, massivi e multiparametrici, aiutati da algoritmi e percorsi informatici; la capacità di incorporare percorsi di analisi multimodale (laboratorio ed imaging) nei campi della consultazione globale (EMR), della patologia terapeutica, dei rapporti tra sub-specialità e medicina generale e della produzione di servizi ad alto valore aggiunto; la capacità di filtrare l'information overload e trasformarla in informazioni sintetiche, efficaci e pratiche [47].

\section{Conflitti di interesse Nessuno.}

Studi condotti su esseri umani e animali L'articolo non contiene alcuno studio eseguito su esseri umani e su animali da parte degli autori.

\section{Bibliografia}

1. Cappelletti P (2010) Medicina di Laboratorio. In: Galzigna L, Plebani M (eds) Trattato Italiano di Medicina di Laboratorio. Volume I. Biochimica Clinica Generale. Piccin Nuova Libraria, Padova

2. Cappelletti P (2004) Preparando Modena. Cos'è e dove va la Medicina di Laboratorio. Riv Med Lab-JLM 5:187-196

3. Price CP (2010) Roots, development and future directions of laboratory medicine. Clin Chem Lab Med 48:903-909

4. Morandini M (2014) Innovazione organizzativa in Medicina di Laboratorio. Riv Ital Med Lab 10:1-5

5. Morandini M (2012) Scenari e ruoli della Medicina di Laboratorio al tempo della crisi: pianificazione, organizzazione e gestione. Riv Ital Med Lab 8:196-207

6. Cappelletti P (2012) Medicina di Laboratorio: un futuro possibile. Riv Ital Med Lab 8:121-130

7. O'Leary JJ (2006) Pathology 2026: The future of laboratory medicine and academic pathology. In: O'Leary JJ (ed) Understanding disease. Wiley, New York

8. Cappelletti P (2012) Scenari e ruoli della Medicina di Laboratorio al tempo della crisi: da Modena a Salerno ed oltre. Riv Ital Med Lab 8:183-189

9. Nanni M (2012) Scenari e ruoli della Medicina di Laboratorio al tempo della crisi: i sacerdoti della tecnologia. Riv Ital Med Lab 8:190-195

10. Cenci AM (2012) Scenari e ruoli della Medicina di Laboratorio al tempo della crisi: consulenza clinica. Riv Ital Med Lab 8:208-215

11. Dorizzi RM, Baldrati L, Vascotto L et al (2012) Scenari e ruoli della Medicina di Laboratorio al tempo della crisi: governance clinica, EBLM, linee guida, PDT/PA. Riv Ital Med Lab $8: 216-230$

12. Plebani M (2015) Clinical laboratories: production industry or medical service? Clin Chem Lab Med 53:995-1004

13. Pradella M (2004) Il flusso operativo (workflow) delle prestazioni di laboratorio. Riv Med Lab_JLM 5(Suppl. 3):105-107

14. Dorizzi RM (2007) Una roadmap per la Information Technology in Medicina di Laboratorio. RIMeL/IJLaM 3:75-81

15. Sorace J, Aberle DR, Eliman D et al (2012) Integrating pathology and radiology disciplines: an emerging opportunity? BMC Med 10:100-105

16. Diamandis EP (2015) Theranos Phenomenon: promises and fallacies. Clin Chem Lab Med 53:989-993
17. Oosterhuis WP, Zerah S (2015) Laboratory Medicine in the European Union. Clin Chem Lab Med 53:5-14

18. AA VV (2001) Point-of-care testing. Riv Med Lab-JLM 2:5-84

19. GdS POCT e GdS EBLM SIMeL (2009) Posizione SIMeL su POCT e diagnostica decentrata: una proposta. RIMeL/IJLaM 5:68-73

20. Felder RA, Graves S, Mifflin T (1999) Reading the future: the increased relevance of laboratory medicine in the next century. MLO Med Lab Obs 31:20-21, 24-26

21. Cappelletti P (2004) Un Patto per la Modernizzazione ed Umanizzazione della Medicina di Laboratorio in Italia. Riv Med Lab_JLM 5:252-254

22. http://www.annenbergpublicpolicycenter.org/Downloads/ Hyperlinked_Society_Article.pdf (Accesso 5 settembre 2015)

23. Van De Belt TH, Engelen LJ, Berber SA et al (2010) Definition of Health 2.0 and Medicine 2.0: a systematic review. J Med Internet Res 12:e18

24. Lindberg DA, Humphreys BL (1998) Medicine and Health on the Internet: the good, the bad, and the ugly. JAMA 280:1303-1304

25. http://www.pewinternet.org/2013/01/15/health-online-2013/ (Accesso 5 settembre 2015)

26. http://hlwiki.slais.ubc.ca/index.php/Social_media_in_health: _history,_movements_and_current_practices (Accesso 5 settembre 2015)

27. Hughes B, Joshi I, Wareham J (2008) Health 2.0 and medicine 2.0: tensions and controversies in the field. J Med Internet Res 10:e23

28. Ozdalga E, Ozdalga A, Ahuja N (2012) The Smartphone in Medicine: a review of current and potential use among Physicians and Students. J Med Internet Res 14:e128

29. https://readytogo.microsoft.com/global/_layouts/RTG/ AssetViewer.aspx?AssetUrl=https\%3A\%2F\%2Freadytogo. microsoft.com\%2Fglobal\%2FAsset\%2FPages \%2FMicrosoft $\%$ 20Advanced\%20Analytics\%20in\%20Health\%20eBook.aspx (Accesso 5 settembre 2015)

30. http://www.cdwcommunit.com/resources/infographic/digitalpatient/ (Accesso 5 settembre 2015)

31. Tecnologia da capo a piedi (2014) Il Sole 24 Ore-Nòva. Domenica 12 gennaio, pag. 12

32. http://www.cdwcommunit.com/perspectives/expert-perspectives/ wearable-technology-smart-fashion/ (Accesso 5 settembre 2015)

33. Sottocorona C (2015) Internet delle cose. Così i piccoli possono diventare grandi. Corriere Economia, 14 settembre, pag. 39

34. http://www.fda.gov/downloads/MedicalDevices/ DeviceRegulationandGuidance/GuidanceDocuments/ UCM263366.pdf (Accesso 5 settembre 2015)

35. Komatireddy R, Topol EJ (2012) Medicine Unplugged: the future of Laboratory Medicine. Clin Chem 58:1644-1647

36. Wood J (2002) The roles, duties and responsabilities of technologists in the clinical laboratory. Clin Chim Acta 319:127-132

37. Diamandis EP (2002) Duties and responsabilities of laboratory scientists. Clin Chim Acta 319:111-115

38. Royal College of Pathologists (2004) The future roles of medical graduates and consultants in pathology services. https:// www.rcpath.org/NR/rdonlyres/1835BF5A-DCD4-478F-A85BBE1DB941BB19/0/medicspathol.pdf (Accesso 5 settembre 2015)

39. Smith BR, Wells A, Alexander CB et al (2006) Curriculum content and evaluation of resident competency in Clinical Pathology (Laboratory Medicine): a proposal. Clin Chem 52:917-949

40. Beastall G, Kenny D, Laitinen P et al (2005) A guide to defining the competence required of a consultant in clinical chemistry and laboratory medicine. Clin Chem Lab Med 43:654-659

41. Cappelletti P (2005) La risposta della Medicina di Laboratorio al quesito clinico. RIMeL/IJLaM 1(Suppl.):15-23

42. Cappelletti P (2008) Come cambia la Medicina di Laboratorio. RIMeL/IJLaM 4:26-30 
43. Goldschmidt MJ (2004) The NEXUS vision: an alternative to the reference value concept. Clin Chem Lab Med 42:868-873

44. Beastall GH (2015) Harmonisation of specialist training and continuing professional development in laboratory medicine: a long but necessary journey. Clin Chem Lab Med 53:1-3

45. Longo F (2011) Scenari per la sanità futura: quale strategia di ruolo per i dirigenti di laboratorio? In: Scenari sulla trasformazione del capitale umano. Workshop Siemens Healthcare Diagnostics. $1^{\circ}$ Congresso Nazionale della Medicina di Laboratorio, Parma. 15-18 novembre 2011
46. Cappelletti P (2014) La complessità e la Medicina di Laboratorio. Riv Ital Med Lab 10:187-192

47. Cappelletti P (2013) Innovazione ed evidenze in Medicina di Laboratorio. Riv Ital Med Lab 9:177-184

48. Scott MG, Rifai N, Smith B et al (2015) The Changing Face of Laboratory Medicine: a more service and less academically oriented profession? Clin Chem 61:322-329 